\title{
An Optical Setup for Deterministic Creation of Four Partite $W$ state
}

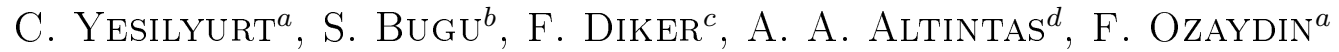 \\ ${ }^{a}$ Isık University, Istanbul, Turkey, ${ }^{b}$ Istanbul University, Istanbul, Turkey \\ ${ }^{c}$ Bogazici University, Istanbul, Turkey, ${ }^{d}$ Okan University, Istanbul, Turkey
}

\begin{abstract}
In order to create polarization based entanglement networks of $W_{4}$ state, we propose an optical setup, which uses only four horizontally polarized photons as resource which implies no entanglement requirement as a resource. This setup can generate target state deterministically, by operating several quantum optical gates, which can be realized with current photonics technology. The setup we propose is composed of one Not, two Hadamard, five Controlled Not (CNot) and one Toffoli gate.
\end{abstract}

DOI: 10.12693/APhysPolA.127.1230

PACS: 03.67.-a, 03.65.Ud, 03.65.Yz

\section{Introduction}

Quantum entanglement plays a vital role for quantum information and quantum computation applications. Non-classical correlation between two or more particles, the entanglement, has attracted attention after EPR paper [1]. Both fundamental quantum features and information theoretical perspective [2] of entanglement have been studied by researchers. Creation, manipulation and quantification of bipartite entanglement has now been understood sufficiently in terms of quantum information tasks [2-6]. Bipartite entangled states can be converted to each other by local operations and classical communication (LOCC), but the same is not true for multipartite entanglement states [7]. Our understanding of multipartite entanglement needs to improve, because some quantum information applications cannot be realized by using only bipartite entanglement and sometimes different class of multipartite entanglement states are required for various tasks, such as GHZ states for achieving consensus in distributed networks [8], $W$ states for leadership election in anonymous quantum networks [9] and cluster states for measurement-based quantum computation, etc. In order to realize such tasks, we need to create a specific quantum state, which has a determined number of particles. The expansion and fusion operations of cluster and GHZ states have already been demonstrate experimentally and theoretically $[10,11]$ but, due to its more complicated structure, creation of $W$ states becomes challenging and an efficient scheme has not been experimentally demonstrated yet. Fusion of two $W$ states or expansion of a $W$ state with ancillary photons have been studied in optic setups [12-18]. Recently by integrating a Fredkin gate into setup from [19], we have increased the success probability of fusion process and increased the size of the final state [20]. With new setups including the basic

corresponding author; e-mail: can--yesilyurt@hotmail.com fusion gate of [19], we achieved simultaneous fusion of four $W$ states [21] and three $W$ states [22]. However all these schemes can generate larger $W$ state probabilistically and need smaller $W$ states as resource. Due to the probabilistic process, resource cost of generated $W$ state increases with respect to size of the final state. In this work we propose an optical setup to create polarization based entanglement $W_{4}$ state. Unlike previous schemes, this method does not require smaller $W$ state as the input state and it can deterministically create $W_{4}$ by using only four horizontally polarized $(\mathrm{H})$ photons. On the other hand, the previous setups have to start the fusion process $W_{3}$ to obtain a $W_{4}$ state. Therefore there is a cost to obtain first $W_{3}$ plus the other cost for obtaining $W_{4}$ from smaller ones. With the proposed here setup one can start with a $W_{4}$ directly, thus bypassing the first and second steps of fusion process, which will decrease the resource cost significantly.

\section{Working process of fusion scheme}

Since the fusion operation with the proposed setup is not probabilistic, no photon is going to be measured, therefore no photon will be lost. Simplest elements, that are used in this scheme are Not and Hadamard gates. These are elementary quantum gates that are used commonly. The mission of CNot gate is to control one of input qubits and rotate by 90 degrees the other ones, if the controlled qubit is vertically polarized $(\mathrm{V})$. It has been realized by means of several technologies such as superconductors [23], ion-trap systems and photonics [24, 25]. Another element which we use to perform the operation of "double controlled not", is called Toffoli gate. It can control two inputs and if both are V-polarized photons, then it performs the Not operation on target qubit. This quantum gate is the most sophisticated one in this setup. Although a hundred percent successfully working Toffoli gate has not been demonstrated by using linear optics, there is a successful proposal with a measurement-based quantum computing, using weighted graph states [26]. 
Fuirasek proposed a linear optic setup for the implementation of Toffoli gate [27]. In superconducting [28] and ion-trap [29] systems, Toffoli gate has already been demonstrated. Working principle of proposed setup is presented in Fig. 1. In this scheme we used CNot gates in steps 2, 3, 5, 6 and 7 (Fig. 1), Toffoli gate in step 4, Hadamard and Not gates in step 1. Four separate Hpolarized photons are sent to spatial modes 1, 2, 3 and 4, then all these quantum optical operations are performed on these photons. In step one, mode 1 and 4 start with Hadamard gate, they convert the $|H\rangle$ to $\frac{|H\rangle+|V\rangle}{\sqrt{2}}$ then, when we operate the CNot gate, a two photon entangled state is generated. The other CNots and Toffoli gates are used to extend the entanglement that we create, due to their arrangement, we can obtain a genuine $W_{4}$ state as a result.

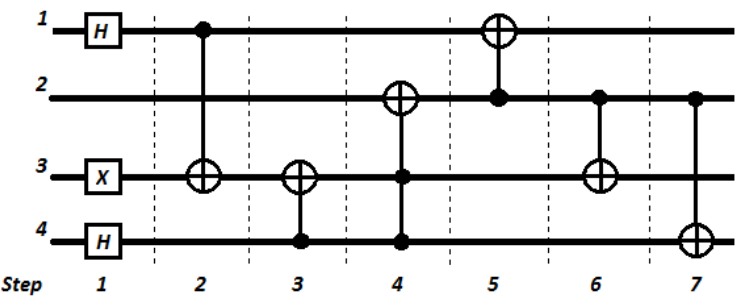

Fig. 1. Proposed optical setup, which can deterministically create $W_{4}$ state. It requires four H-polarized photons for sending to lines 1-4, and three single qubit operations are performed in step 1 and 5 two qubit gates operate in step 2, 3, 5, 6 and 7 . Only one three-qubit Toffoli gate is used in step 4 .

One of the important things is that, cost of prepared $W$ state should be as low as possible. For instance, in probabilistic systems, in order to reach a target size $W$ state, many smaller size $W$ states can be used as resources and system has to work many times. This setup performs the creation operation with unit success probability, without using any entanglement state as a resource. These are main advantages, which reduce the cost of target $W$ state. On the other hand, main drawback of proposed method is that, it can create only $W_{4}$, but it may be modified for target size of $\mathrm{W}$ states by adding several (two or three) qubit gates.

In the generation process, four parties, Alice, Bob, Charlie and Dave have one H-polarized photon each, $|H\rangle_{A},|H\rangle_{B},|H\rangle_{C}$ and $|H\rangle_{D}$, respectively, and they want to create a $W_{4}$ by using their photons. The target state that they wish to reach can be written as

$$
\begin{aligned}
& \left|W_{4}\right\rangle=\frac{1}{\sqrt{4}}\left(\left|H_{A} H_{B} H_{C} V_{D}\right\rangle+\left|H_{A} H_{B} V_{C} H_{D}\right\rangle\right. \\
& \left.\quad+\left|H_{A} V_{B} H_{C} H_{D}\right\rangle+\left|V_{A} H_{B} H_{C} H_{D}\right\rangle\right) .
\end{aligned}
$$

The creation gates, as shown in Fig. 1, take their photons from lines and perform all shown quantum optical operations. The concept of generation is shown in Fig 2 .

Now, parties put together their photons, the whole state as follows,

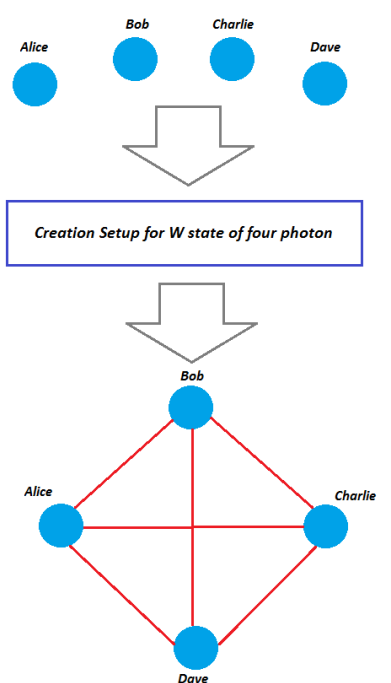

Fig. 2. Schematic illustration of creation of $W$ state. Four Horizontal polarized photons are sent to the creation setup presented in Fig.1, after the operations, $W$ state of four photon is created. The edges connecting the qubits represents the complex entanglement structure.

$$
|\Psi\rangle=\left|H_{A} H_{B} H_{C} H_{D}\right\rangle .
$$

The creation setup start to perform the operation step by step, in step 1 ,

$$
|\Psi\rangle=\frac{\left|H_{A}\right\rangle+\left|V_{A}\right\rangle}{\sqrt{2}}\left|H_{B}\right\rangle\left|V_{C}\right\rangle \frac{\left|H_{D}\right\rangle+\left|V_{D}\right\rangle}{\sqrt{2}},
$$

which is,

$$
\begin{aligned}
& |\Psi\rangle=\frac{\left|H_{A}\right\rangle\left|H_{B}\right\rangle\left|V_{C}\right\rangle+\left|V_{A}\right\rangle\left|H_{B}\right\rangle\left|V_{C}\right\rangle}{\sqrt{2}} \\
& \quad \times \frac{\left|H_{D}\right\rangle+\left|V_{D}\right\rangle}{\sqrt{2}} .
\end{aligned}
$$

So far, thanks to the Hadamard gate, Alice's and Dave's photons transform the superposition of $|H\rangle$ and $|V\rangle$. Now the CNot gates are taking on a task to distribute the entanglement, which we create. In step two,

$$
\begin{aligned}
& |\Psi\rangle=\frac{\left|H_{A}\right\rangle\left|H_{B}\right\rangle\left|V_{C}\right\rangle+\left|V_{A}\right\rangle\left|H_{B}\right\rangle\left|H_{C}\right\rangle}{\sqrt{2}} \\
& \quad \times \frac{\left|H_{D}\right\rangle+\left|V_{D}\right\rangle}{\sqrt{2}},
\end{aligned}
$$

which is,

$$
\begin{gathered}
|\Psi\rangle=\frac{1}{\sqrt{2}}\left(\left|H_{A}\right\rangle\left|H_{B}\right\rangle \frac{\left|V_{C}\right\rangle\left|H_{D}\right\rangle+\left|V_{C}\right\rangle\left|V_{D}\right\rangle}{\sqrt{2}}\right. \\
\left.+\left|V_{A}\right\rangle\left|H_{B}\right\rangle \frac{\left|H_{C}\right\rangle\left|H_{D}\right\rangle+\left|H_{C}\right\rangle\left|V_{D}\right\rangle}{\sqrt{2}}\right) .
\end{gathered}
$$

In step three, the other CNot from Dave's photon to Charlie's.

$$
\begin{gathered}
|\Psi\rangle=\frac{1}{\sqrt{2}}\left(\left|H_{A}\right\rangle\left|H_{B}\right\rangle \frac{\left|V_{C}\right\rangle\left|H_{D}\right\rangle+\left|H_{C}\right\rangle\left|V_{D}\right\rangle}{\sqrt{2}}\right. \\
\left.+\left|V_{A}\right\rangle\left|H_{B}\right\rangle \frac{\left|H_{C}\right\rangle\left|H_{D}\right\rangle+\left|V_{C}\right\rangle\left|V_{D}\right\rangle}{\sqrt{2}}\right),
\end{gathered}
$$

which is, 


$$
\begin{aligned}
& |\Psi\rangle=\frac{1}{\sqrt{4}}\left(\left|H_{A}\right\rangle\left|H_{B}\right\rangle\left|V_{C}\right\rangle\left|H_{D}\right\rangle\right. \\
& +\left|H_{A}\right\rangle\left|H_{B}\right\rangle\left|H_{C}\right\rangle\left|V_{D}\right\rangle \\
& +\left|V_{A}\right\rangle\left|H_{B}\right\rangle\left|H_{C}\right\rangle\left|H_{D}\right\rangle \\
& \left.+\left|V_{A}\right\rangle\left|H_{B}\right\rangle\left|V_{C}\right\rangle\left|V_{D}\right\rangle\right) .
\end{aligned}
$$

Now, Toffoli gate performs Not operation to line 2 by controlling line 3 and 4 in step four,

$$
\begin{aligned}
& |\Psi\rangle=\frac{1}{\sqrt{4}}\left(\left|H_{A}\right\rangle\left|H_{B}\right\rangle\left|V_{C}\right\rangle\left|H_{D}\right\rangle\right. \\
& +\left|H_{A}\right\rangle\left|H_{B}\right\rangle\left|H_{C}\right\rangle\left|V_{D}\right\rangle \\
& +\left|V_{A}\right\rangle\left|H_{B}\right\rangle\left|H_{C}\right\rangle\left|H_{D}\right\rangle \\
& \left.+\left|V_{A}\right\rangle\left|V_{B}\right\rangle\left|V_{C}\right\rangle\left|V_{D}\right\rangle\right) .
\end{aligned}
$$

Here, our results start to be clear, step five, six and seven, CNots are finishing the mission of setup and transform the state to a $W_{4}$

$$
\begin{aligned}
& |\Psi\rangle=\frac{1}{\sqrt{4}}\left(\left|H_{A}\right\rangle\left|H_{B}\right\rangle\left|V_{C}\right\rangle\left|H_{D}\right\rangle\right. \\
& \quad+\left|H_{A}\right\rangle\left|H_{B}\right\rangle\left|H_{C}\right\rangle\left|V_{D}\right\rangle \\
& +\left|V_{A}\right\rangle\left|H_{B}\right\rangle\left|H_{C}\right\rangle\left|H_{D}\right\rangle \\
& \left.+\left|H_{A}\right\rangle\left|V_{B}\right\rangle\left|H_{C}\right\rangle\left|H_{D}\right\rangle\right) .
\end{aligned}
$$

\section{Conclusions}

We have introduced an optical gate which consists of one Toffoli, two Hadamard, one Not and five CNot gates. The proposed setup does not only deterministically creates $W$ state of four photons but also, it reduces the cost of the target $W$ state for previous setups that take the ready $W$ state of four photons from this setup. From the experimental perspective, since the process is deterministic, only one run is enough to create a $W$ state of four photons. This setup can be efficiently demonstrated with current photonics technology using measurementbased one way quantum compuation.

\section{References}

[1] A. Einstein, B. Podolsky, N. Rosen, Phys. Rev. 47, 777 (1935).

[2] R. Horodecki, P. Horodecki, M. Horodecki, K. Horodecki, Rev. Mod. Phys. 81, 865 (2009).

[3] N. Gisin, G. Ribordy, W. Tittel, H. Zbinden, Rev. Mod. Phys. 74, 145 (2002).

[4] V. Giovanetti, S. Lloyd, L. Maccone, Phys. Rev. Lett. 96, 010401 (2006).

[5] H.J. Kimble, Nature 453, 1023 (2008).
[6] T.D. Ladd, F. Jelezko, R. Laflamme, Y. Nakamura, C. Monroe, J.L. O'Brien, Nature 464, 45 (2010).

[7] W. Dür, G. Vidal, J.I. Cirac, Phys. Rev. A. 62, 062314 (2000).

[8] D.M. Greenberger, M. Horne, A. Shimony, A. Zeilinger, Am. J. Phys. 58, 1131 (1990).

[9] E. D'Hondt, P. Panangaden, Quantum Inf. Comput. 6, 173 (2006).

[10] D.E. Browne, T. Rudolph,, Phys. Rev. Lett. 95, 010501 (2005).

[11] A. Zeilinger, M.A. Horne, H. Weinfurter, M. Zukowski, Phys. Rev. Lett. 78, 3031 (1997).

[12] T. Tashima, T. Wakatsuki, S.K. Ozdemir, T. Yamamoto, M. Koashi, N. Imoto, Phys. Rev. Lett. 102, 130502 (2009).

[13] T. Tashima, S.K. Ozdemir, T. Yamamoto, M. Koashi, N. Imoto, Phys. Rev. A 77, 030302(R) (2008).

[14] T. Tashima, S.K. Ozdemir, T. Yamamoto, M. Koashi, N. Imoto, New. J. Phys. 11, 023024 (2009).

[15] T. Tashima, T. Kitano, S.K. Ozdemir, T. Yamamoto, M. Koashi, N. Imoto, Phys. Rev. Lett. 105, 210503 (2010).

[16] Y. Li, T. Kobayashi, , Phys. Rev. A 70, 014301 (2004).

[17] M. Eibl, N. Kiesel, M. Bourennane, C. Kurtsiefer, H. Weinfurther, Phys. Rev. Lett. 92, 077901 (2004).

[18] H. Mikami, Y. Li, K. Fukuoka, T. Kobayashi, Phys. Rev. Lett. 95, 150404 (2005).

[19] S.K. Ozdemir, E. Matasunga, T. Tashima, T. Yamamoto, M. Koashi, N. Imoto, New. J. Phys. 13, 103003 (2011).

[20] S. Bugu, C. Yesilyurt, F. Ozaydin, Phys. Rev. A 87, 032331 (2013).

[21] C. Yesilyurt, S. Bugu, F. Ozaydin, Quantum Inf. Process. 12, 2965 (2013).

[22] F. Ozaydin, S. Bugu, C. Yesilyurt, A.A. Altintas, M. Tame, S.K. Ozdemir, Phys. Rev. A 89, 042311 (2014).

[23] J.H. Plantenberg, P.C. de Groot, C.J.P.M. Harmans, J.E. Mooji, Nature 447, 836 (2007).

[24] R. Okamoto, H.F. Hofmann, S. Takeuchi, K. Sasaki , Phys. Rev. Lett. 95, 210506 (2005).

[25] N. Kiesel, C. Schmid, U. Weber, R. Ursin, H. Weinfurter, Phys. Rev. Lett. 95, 210505 (2005).

[26] M.S. Tame, S.K. Ozdemir, M. Koashi, N. Imoto, M.S. Kim, Phys. Rev. A 79, 020302(R) (2009).

[27] J. Fuirasek, Phys. Rev. A 73, 062313 (2006).

[28] A. Fedorov, L. Steffen, M. Baur, M.P. da Silva, A. Wallraff, Nature 481, 170 (2012).

[29] T. Monz, K. Kim, W. Hänsel, M. Riebe, A.S. Villar, P. Schindler, M. Chwalla, M. Hennrich, R. Blatt, Phys. Rev. Lett. 102, 040501 (2009). 